\title{
LA INCIDENCIA DE LOS HORMIGONES DE CEMENTO ALUMINOSO EN MADRID
}

\author{
(THE CONSEQUENCES OF THE USE OF CONCRETES WITH HIGH ALUMINA CEMENT \\ IN MADRID)
}

Fernando Macías Hidalgo-Saavedra, Dr. Arquitecto

Jefe del Dpto. de Protección de la Edificación de Gerencia Municipal de Urbanismo

Ayuntamiento de Madrid

ESPAÑA

Fecha de recepción: 9.XII-92

615.11

\section{RESUMEN}

En este articulo se presenta, en primer lugar, el planteamiento seguido por el Ayuntamiento de Madrid para el estudio de viviendas construidas con cemento aluminoso, comparando el caso de Madrid con el de otros municipios españoles. Se incluye, además, un caso concreto: el del Estadio Vicente Calderón, en Madrid, donde se indican las actuaciones seguidas tras detectar cemento aluminoso en las viguetas prefabricadas pretensadas de los forjados inclinados que soportan las graderías del estadio.

\section{SUMMARY}

This article presents, in the first place, the idea coming from the Madrid Town Council (Ayuntamiento de Madrid) to study the dwellings built with high alumina cement, comparing the case of Madrid with those of other Spanish municipalities. The article also includes one particular case, that of the Vicente Calderón Stadium (Madrid) where we can see what was done after the detection of high alumina cement in prestressed precast beams of the inclined slabs which supported the tiers of the stadium.

\section{UN PRIMER APUNTE}

En el proceso de deterioro del hormigón armado inciden, de manera importante, dos tipos de mecanismos:

1.-De agresión al hormigón, y

2. - De agresión a la armadura.

Entre los del primer grupo se destacan:

a) Las reacciones álcali-sílice.

b) Ataques químicos, externos-internos.

c) Alteraciones por ciclos hielo/deshielo.

Entre los del segundo grupo, la corrosión destruye la armadura de forma progresiva e irremediable.

En ambos grupos, la presencia del agua y de la sal, son parámetros determinantes.
Pero cuando en la masa del hormigón armado está presente el cemento aluminoso, existen factores que aceleran el proceso de "conversión", siendo los más importantes:

- Temperatura.

- Humedad.

- Relación agua-cemento.

- Alcalinidad de la fase acuosa intersticial.

Pues bien, es en estos factores donde encontramos las diferencias entre el problema de la degradación del cemento aluminoso en el ámbito de Madrid y la situación en otras zonas donde ya han sufrido, y aún sufren, estos daños (Barcelona, Canarias, Sevilla, etc.).

- La temperatura media de Madrid es más baja que la de las zonas del litoral mediterráneo.

- La humedad relativa es sensiblemente inferior. 
- El nivel medio de construcción de los años 50 a 70, en Madrid, se presenta superior al de otras regiones, siendo de aceptable a bueno.

Este primer apunte de la situación no debe hacernos olvidar la trascendencia de microclimas en nuestros edificios -en las zonas "húmedas"- de vicios ocultos, tanto por la manipulación de elementos parciales de la estructura (viguetas de forjados), como por su puesta en obra y el deterioro que produce la falta de una cultura de conservación de las viviendas, que acostumbren al usuario al periódico control del estado de sus instalaciones y estructuras.

Toda vez que tenemos tiempo, antes de que el deterioro del cemento aluminoso en nuestros forjados presente carácteres irreversibles, empleémoslo a nuestro favor.

\section{INICIO Y JUSTIFICACIÓN DEL ESTUDIO}

El Ayuntamiento de Madrid, a raíz del accidente ocurrido en viviendas del Turó de la Peira, en Barcelona, (11/11/90), por la degradación de los elementos de construcción con cemento aluminoso, ordenó el oportuno estudio de esta nueva patología, sus antecedentes, incidencias y soluciones, así como su posible evolución en el término municipal de Madrid.

Ante la magnitud del problema, se crearon 3 frentes de estudio, con objeto de conocer:

A) Características técnicas de estos aglomerantes y proceso de transformación. Agentes que actúan durante el proceso e incidencia en el tiempo.

B) Experiencias sufridas y soluciones adoptadas en otros municipios nacionales o internacionales.

C) Localización del problema en Madrid, situación actual del proceso patológico y propuesta de actuación.

Igualmente se señalaron pautas de comportamiento para los equipos investigadores:

- Discreción para no crear una situación precipitada de angustia o preocupación en los administrados.
- Máxima amplitud de fuentes de información:

- Colegios profesionales.

- Agrupaciones de cementeros.

- Generalitat de Cataluña.

- Ayuntamiento de Barcelona.

- MOPT.

- Laboratorios especializados.

- Publicaciones sobre el tema.

- Archivos de patología propios.

- Etc.

- Consideración de "vivo en el tiempo" del estudio, completándolo periódicamente con los nuevos conocimientos que se hayan obtenido.

- Mantener abierta la "Propuesta de Actuación", en espera del estudio de la patología en nuestro área de actuación del marco jurídico, económico y social que se adopte por la Administración Central, y de la respuesta de las Administraciones Autónomicas en aquellas Autonomías (Canarias y Cataluña) donde ya han padecido el problema.

El trabajo es encomendado al Departamento de Protección de la Edificación, de Gerencia Municipal de Urbanismo, que redacta un primer informe en febrero de 1992, siendo en marzo cuando, de manera formal, el Ayuntamiento solicita de la Comunidad Autónoma de Madrid y del MOPT las mismas ayudas económicas concertadas que ya existen en otras regiones.

El Ayuntamiento organiza la "PRIMERA JORNADA SOBRE PATOLOGÍAS Y DEGRADACIONES DEL HORMIGÓN", el 6 de mayo de 1992. A través de su Arquitecto, Jefe de la Sección de Edificación Deficiente, participa en las "JORNADAS SOBRE CEMENTO ALUMINOSO", en el Instituto de Ciencias de la Construcción Eduardo Torroja, los días 4 y 5 de junio, colabora con los Colegios de Arquitectos y de Arquitectos Técnicos y Aparejadores, en sendas mesas redondas y, por último, patrocina la exposición de SOLUCIONES PARA FORJADOS DE VIGUETAS DE HORMIGÓN: "ALUMINOSIS" desde el 16 de junio, hasta el 16 de julio, incluyendo jornadas técnicas, coloquios y conferencias dentro del programa.

En la actualidad, conjuntamente con representantes del MOPT y de la CAM, se estudian y cuantifican las ayudas económicas necesarias de fondos públicos, para abordar el problema creado por el proceso del cemento aluminoso en nuestra región. 


\section{LA SITUACIÓN EN MADRID}

En una primera aproximación para definir los parámetros del problema en Madrid, partiremos del cuadro de producción de cemento aluminoso en España, su consumo conocido en Cataluña y remanente para el resto de España.

PRODUCCIÓN Y CONSUMO DE CEMENTO ALUMINOSO EN CATALUÑNA Y RESTO DE ESPAÑA

\begin{tabular}{|c|c|c|c|}
\hline AÑo & PRODUCCION C.A. & $\begin{array}{l}\text { CONSUMO } \\
\text { CATALUÑNA }\end{array}$ & $\begin{array}{c}\text { RESTO } \\
\text { ESPAÑ̃A }\end{array}$ \\
\hline 1950 & 2.192 & 1.589 & 603 \\
\hline 1951 & 6.194 & 3.040 & 3.154 \\
\hline 1952 & 9.609 & 5.952 & 3.757 \\
\hline 1953 & 15.406 & 9.402 & 6.004 \\
\hline 1954 & 16.861 & 10.229 & 5.432 \\
\hline 1955 & 23.065 & 14.117 & 8.948 \\
\hline 1956 & 26.028 & 21.348 & 4.680 \\
\hline 1957 & 31.442 & 19.648 & 11.794 \\
\hline 1958 & 30.452 & 20.071 & 10.381 \\
\hline 1959 & 32.017 & 20.449 & 11.568 \\
\hline 1960 & 33.602 & 19.655 & 13.947 \\
\hline 1961 & 38.410 & 21.898 & 16.512 \\
\hline 1962 & 44.356 & 26.640 & 17.716 \\
\hline 1963 & 55.035 & 32.075 & 22.960 \\
\hline 1964 & 59.134 & 38.475 & 20.659 \\
\hline 1965 & 62.051 & 38.605 & 23.446 \\
\hline 1966 & 62.127 & 34.133 & 27.994 \\
\hline 1967 & 59.662 & 25.078 & 34.584 \\
\hline 1968 & 55.491 & 19.883 & 35.608 \\
\hline 1969 & 61.654 & Sin datos & Sin datos \\
\hline 1970 & 59.181 & Sin datos & Sin datos \\
\hline TOTAL & $783.969 \mathrm{t}$ & $382.297 t$ & $401.672 \mathrm{t}$ \\
\hline $\begin{array}{l}\text { TOTAL } \\
\text { PONDERADO } \\
\text { SIN 1969/ }\end{array}$ & & & \\
\hline 1970 & $663.134 \mathrm{t}$ & $382.297 \mathrm{t}$ & $280.837 \mathrm{t}$ \\
\hline PORCENTAJE & $100 \%$ & $57 \%$ & $43 \%$ \\
\hline
\end{tabular}

La primera conclusión nos indica que el consumo de cemento aluminoso, en Cataluña, supera al de las demás Comunidades juntas, alcanzando el $57 \%$ de la producción final.

Debe tenerse en cuenta que, dadas las características técnicas del producto, se empleó en construcciones civiles, presas, puertos, edificios industriales, escolares, etc.
Ya en las PRIMERAS JORNADAS TÉCNICAS SOBRE DURABILIDAD DE LOS HORMIGONES, señala Sánchez Pirez:

"Ciñéndonos al aspecto práctico del empleo del cemento aluminoso en los terrenos yesíferos, vamos a destacar:

- FECSA lo emplea desde hace más de 40 años.

- Salto de San Lorenzo, 1928/1929.

- Salto de la Plana, 1930.

- Salto de Flix, entre 1941/1946.

- Salto de Balaguer, entre 1954/1959.

Hasta la fecha, en ninguno de ellos, se ha apreciado anormalidad alguna.

La sociedad Electro Metalúrgica del Ebro, en el Salto de Menuza, 1958, ha registrado igualmente resultados muy satisfactorios.

Lo mismo cabe decir de la Sociedad Productora de Fuerzas Motrices, S.A., en su Salto del Valle de Arán.

El cemento fundido se ha empleado en el Canal de los Monegros, en revestimiento de hormigón en masa, en revestimientos con cañón lanza-cemento para impermeabilización, y en cimentación de las pilas del acueducto de Tardienta.

En el Canal de Lodosa, se empleó el cemento aluminoso (1933 y 1934) en el revestimiento de un túnel excavado en terreno muy yesoso, después de haber tenido que abandonar una parte del Canal construido con cemento portland, debido al ataque de sulfatos.

El cemento aluminoso se empleó también en revestimientos de hormigón en masa en diversos trozos del canal a cielo abierto, en zonas de yesos.

Otro ejemplo típico, de la influencia que el trato dado al cemento aluminoso puede ejercer en los resultados de una obra, lo tenemos en el caso ocurrido en la construcción del ferrocarril de Teruel a Alcañiz.

Al poco tiempo de empezar la construcción de las obras del IV tramo, se observó que las cimentaciones eran atacadas en el tramo entre Alcañiz y Calanda por los yesos y aguas subálveas agresivas, llegándose en algunos casos a extraer los hormigones de las zanjas de cimentación en estado francamente plástico, después de un endurecimiento aparentemente normal. 
Se dispuso entonces, por la Administración, emplear en los hormigones cemento aluminoso, pero como su precio era aproximadamente doble que el de portland, se redujo la dosificación a $100 \mathrm{~kg} / \mathrm{m}^{2}$ en las cimentaciones y alzados, y a $125 \mathrm{~kg} / \mathrm{m}^{2}$ en las bóvedas, evitándose así el encarecimiento de los precios unitarios".

En consecuencia, no es aventurado suponer un porcentaje variable desde el $25 \%, 50 \%$ y $75 \%$ de cemento aluminoso en construcción de viviendas quedando, en su caso, un $75 \%$, $50 \%$ ó $25 \%$ para otro tipo de obras.

En base a estos porcentajes y al consumo ponderado para el resto de España, excluido el de Cataluña, que se elevaba a $280.837 \mathrm{t}$, obtenemos:

$\begin{array}{cccc}\text { Porcentaje C.A. } & 25 \% & 50 \% & 75 \% \\ & 70.209 \mathrm{t} & 140.419 \mathrm{t} & 210.627 \mathrm{t}\end{array}$

Suponiendo para el ámbito territorial del municipio de Madrid un consumo entre el 10 y el $20 \%$ del total para el resto de España, tomamos el supuesto mayor (20\%), dándonos un consumo en elementos constructivos de viviendas del orden:

$$
14.041 \mathrm{t} \quad 28.083 \mathrm{t} \quad 42.125 \mathrm{t}
$$

Aplicando estas cantidades a las hipótesis elaboradas por el Arquitecto Técnico D. Juan Ramón Rosell (anexo 3), que obtiene 7,35 kilos de cemento aluminoso por $\mathrm{m}^{2}$ de vivienda, situamos un parque de viviendas acorde con cada porcentaje:

\section{SUPERFICIE DE VIVIENDAS}

$$
\text { N. . VIVIENDAS }
$$

PORCENTAJE

A) Viviendas de $100 \mathrm{~m}^{2}$$$
19.100
$$

Viviendas de $100 \mathrm{~m}^{2}$

38.200

$50 \%$

Viviendas de $100 \mathrm{~m}^{2}$

57.310

$75 \%$

B) Viviendas de $70 \mathrm{~m}^{2}$

27.280

$25 \%$

Viviendas de $70 \mathrm{~m}^{2}$

54.570

Viviendas de $70 \mathrm{~m}^{2}$

81.817

$50 \%$

$75 \%$

C) Viviendas de $50 \mathrm{~m}^{2}$

38.200

$25 \%$

Viviendas de $50 \mathrm{~m}^{2}$

76.400

$50 \%$

Viviendas de $50 \mathrm{~m}^{2}$

104.620
En consecuencia, podemos suponer que en Madrid existen alrededor de un parque de 50.000 viviendas, edificadas entre 1950 y 1970 , que total o parcialmente tienen viguetas fabricadas con cemento aluminoso en su estructura.

\section{CONCLUSIONES Y PROPUESTAS}

Como resumen de todo lo hasta ahora estudiado y conocido, aplicado al término municipal de Madrid, podemos decir:

1. El cemento aluminoso está presente en nuestro municipio, siendo su cuantificación entre 20.000 a 50.000 viviendas, repartidas dentro de la autonomía de Madrid.

2. El problema que origina su "conversión", con cambio de cristalización de hexagonal a cúbica, de metaestable a estable, con sensible aumento de porosidad y pérdida de capacidad de trabajo, no presenta en nuestro municipio un grado de peligrosidad similar a la detectada en las zonas costeras, por ser nuestro medio ambiente más seco y, por tanto, con menor grado de humedad relativa.

3. Al día de la fecha se tiene constancia de la existencia no sólo de viviendas, sino también de edificios culturales, dotacionales e industriales, construidos con forjados total o parcialmente, de hormigón con cemento aluminoso.

4. En los casos estudiados por los servicios técnicos del Ayuntamiento de Madrid, con ensayos realizados por laboratorios especializados, sólo se ha detectado una situación peligrosa o alarmante a corto plazo en un edificio (Estadio Vicente Calderón).

5. En las pruebas y ensayos realizados in situ y posteriormente en laboratorios sobre viguetas de hormigón con cemento aluminoso, localizadas en Madrid, la situación general es de "conversión" finalizada, o casi total, pero sin corrosión de las armaduras.

6. Las pruebas de compresión y de límites elásticos dan capacidades residuales de trabajo aún tranquilizadoras, salvo en casos de "micro climas" accidentales (cuartos de calderas, etc.). 
7. Aprovechando las experiencias sufridas en otras ciudades, pretendemos localizar y conocer el problema de Madrid, en labor preventiva que nos permita soluciones no traumáticas para nuestros administrados.

Estamos pues, insisto, en una etapa de localización, evaluación y cuantificación económica del problema que, con una óptica previsora hacia el futuro, nos permita destinar personal cualificado y recursos públicos en beneficio de los administrados.

No obstante, ante la magnitud del problema, y como labor preventiva, conviene proponer:

A) Solicitar la creación de un cuerpo especializado de técnicos que representen a la Administración Central, Autonómica y local, con capacidad para localizar, diagnosticar y proponer soluciones, cuando se conozca la dimensión real del problema que estudiamos.

B) Creación de un marco jurídico adecuado que, contemplando las responsabilidades de los técnicos en la labor de recuperación de estructuras, garantice los aspectos sociales y las diferentes alternativas según la capacidad económica del usuario.

C) Fomentar la cultura de conservación y mantenimiento de la vivienda con campañas institucionales, llamando a participar a todos los que de alguna manera se benefician de este bien.

D) Dar rápida respuesta a la demanda de la sociedad de una Ley de la Construcción que, garantizando los derechos de los futuros usuarios, señale deberes y responsabilidades de todos los integrantes del hecho arquitectónico.

E) Estudiar, hacia el futuro, la creación de un equipo técnico multidisciplinar para el estudio de nuevas patologias del hormigón o de otros materiales, entendiendo que no son eternos y que es aconsejable la inversión en prevención, antes que la reacción ante los siniestros.

\section{UN CASO CONCRETO: ESTADIO VICENTE CALDERÓN - MADRID}

Desde el 26-6-92, se conoce por los servicios técnicos de arquitectura del Club Atlético de Madrid, entidad propietaria del mencionado estadio, el resultado del dictamen elaborado por el Instituto Técnico INTEMAC, encargado al efecto, que confirma la existencia de cemento aluminoso en las viguetas prefabricadas pretensadas de los forjados inclinados que soportan los graderíos del estadio.

El edificio, con planta sensiblemente rectangular de $250 \times 200$ metros lineales, presenta una estructura de pórticos de hormigón armado, ortogonales a los bordes del terreno de juego, sobre los que se apoyan en dos distintos niveles forjados inclinados, realizados en su día por el sistema SAHE de molde metálico recuperable.

La separación media entre pórticos es de $6,00 \mathrm{~m}$, excepto en las zonas de transición entre los diferentes lados del campo, que se efectúa mediante palmeo estructural.

Los forjados inclinados están formados por semiviguetas de hormigón pretensado, complementadas con hormigón vertido en obra que conforma la parte superior de la vigueta y la losa. En los vanos de luces grandes existen nervios transversales, hormigonados in situ, dispuestos a tercios de la luz de las viguetas.

Visualmente se detecta:

- Desprendimientos del hormigón de recubrimiento en las semiviguetas de cemento aluminoso (foto 1).

- Alambres de pretensado totalmente corroído (foto 2).

- Alambres de pretensado con pérdida parcial de sección por corrosión.

- Manchas de óxido férrico.

- Alteración de color característico, en los hormigones de las semiviguetas.

En consecuencia, y ante las órdenes emanadas de las Administraciones actuantes, Delegación del Gobierno y Ayuntamiento de Madrid, se procede a la adopción de medidas de seguridad, bajo la dirección técnica del arquitecto D. Eugenio Sánchez Moro, Jefe de los Servicios de Arquitectura del Club Atlético de Madrid, y de D. José Luis Cano Muñoz, Ingeniero de Caminos.

A petición de la entidad propietaria de las instalaciones, los Servicios Técnicos Municipales asesoran y supervisan los sistemas de seguridad que se estudian y emplean. 


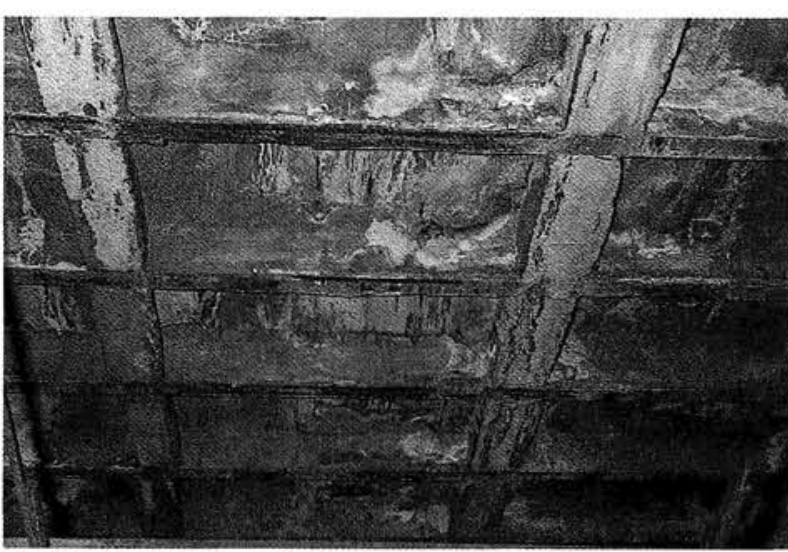

Foto 1.-Situación general del graderío del cuerpo volado.

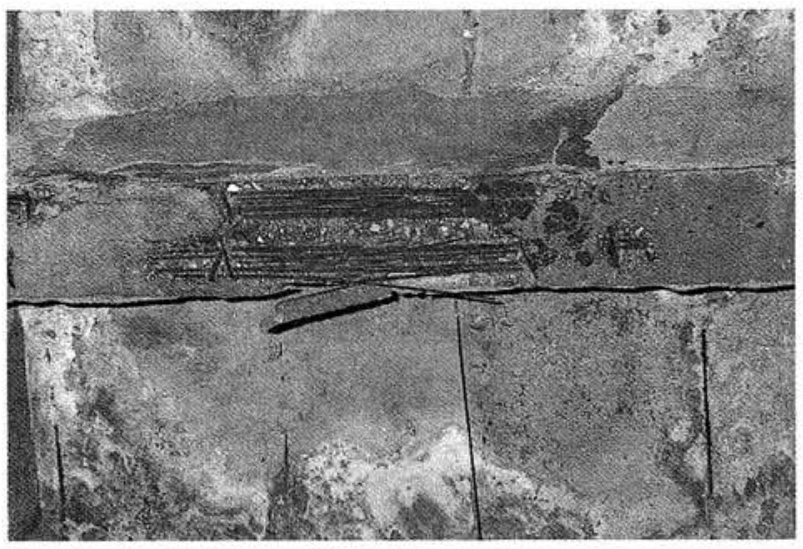

Foto 2.-Detalle de corrosión de las armaduras.

En función de la intensidad de las patologías observadas, se distinguen tres zonas en el estadio que coinciden con la situación de los ambientes más agresivos (humedad y temperatura).

\section{Zona I}

Se corresponde con el lateral oeste, donde está situada la tribuna principal. Coincide con la zona cubierta del estadio.

El estado general del forjado es bueno con mínimos defectos apreciables.

\section{Zona II}

Lateral este y ambos fondos en general, con defectos localizados de distribución aleatoria y con deterioros subsanables.

Desprendimientos parciales de hormigón de protección de las armaduras.

Corrosión del alambre, de acusado a total.

Manchas profusas de óxido férrico.

๑) Consejo Superior de Investigaciones Científicas

Licencia Creative Commons 3.0 España (by-nc)

\section{Zona III}

Dentro del lateral este, y en ambos fondos, sectores que corresponden a la situación de falsos techos y que originan cámaras húmedas de acusada condensación. Notable proceso de corrosión de las armaduras y patología generalizada en todas las viguetas.

Situación alarmante con claro proceso degenerativo irreversible.

\section{ACTUACIÓN EN MEDIDAS DE SEGURIDAD}

Se definen tres líneas de actuación en medidas de seguridad, coincidentes con las distintas zonas anteriormente definidas:

\section{Zona I .}

Ante la situación expuesta, y no pareciendo necesario refuerzo alguno, se realizan tres pruebas de carga de $500 \mathrm{~kg} / \mathrm{m}^{2}$, con resultado satisfactorio.

Se realizarán nuevas inspecciones y, de ser necesario, pruebas de carga cada tres meses como mínimo.

\section{Zona II}

Se plantea una solución de apeo para incrementar su capacidad de trabajo mediante sistema alterno y mediante apoyo elástico con cableado tipo Goliat de siete alambres anclado en vigas, zuncho o vigas ménsula, con apoyos en los zoquetes de unión entre las viguetas de cemento aluminoso.

Se evita la actuación directa en dichas viguetas para no causar nuevas agresiones y pérdida de capacidad residual de trabajo en las mismas.

\section{Zona III}

Se plantea una solución de apeo intensivo (todas las viguetas), incrementando el coeficiente de seguridad y su capacidad de trabajo, incluso planteando la rotura frágil de las viguetas con el sistema ya descrito en el apartado anterior (foto 3).

\section{SOLUCIÓN DE APEO}

El apeo se resuelve mediante un postensado inferior de las viguetas, creando bajo ellas un apoyo elástico que, incremetando la seguridad de los forjados, disminuyen los momentos flectores y cortantes (foto 4).

http://informesdelaconstruccion.revistas.csic.es 


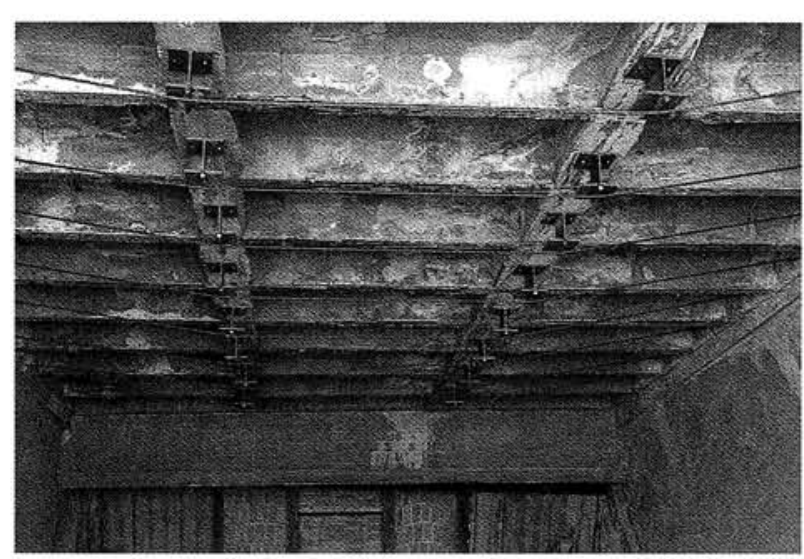

Foto 3.-Vista general del refuerzo.

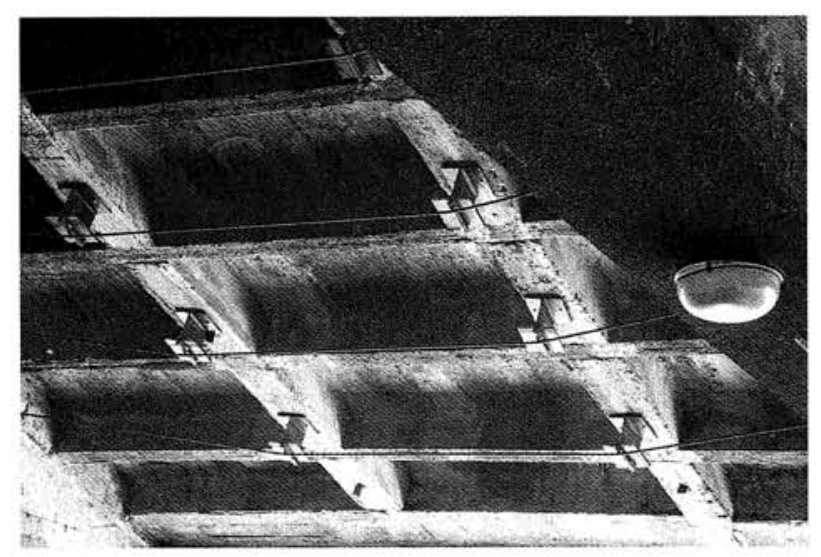

Foto 4.-Detalle de refuerzo.

Esta solución permite controlar las acciones introducidas, variando la tensión y el número de cables que configuran el apoyo elástico (foto 5).

El apoyo inferior se consigue con piezas de perfiles normalizados, dada la urgencia de la actuación, con fijaciones especiales tipo Hilti (foto 6).

\section{MEMORIA DE CÁLCULO}

Entre peso propio del forjado, graderíos y sobrecarga de uso, incluso efectos dinámicos, consideramos un total de $900 \mathrm{~kg} / \mathrm{m}^{2}$.

- Características de los materiales, en medidas de seguridad.

- Hormigón de los forjados fck $=175 \mathrm{~kg} / \mathrm{cm}^{2}$.

- Acero de anclajes y estructural A426.

- Acero de postensar:

- Cordón Goliat super de 7 alambres.

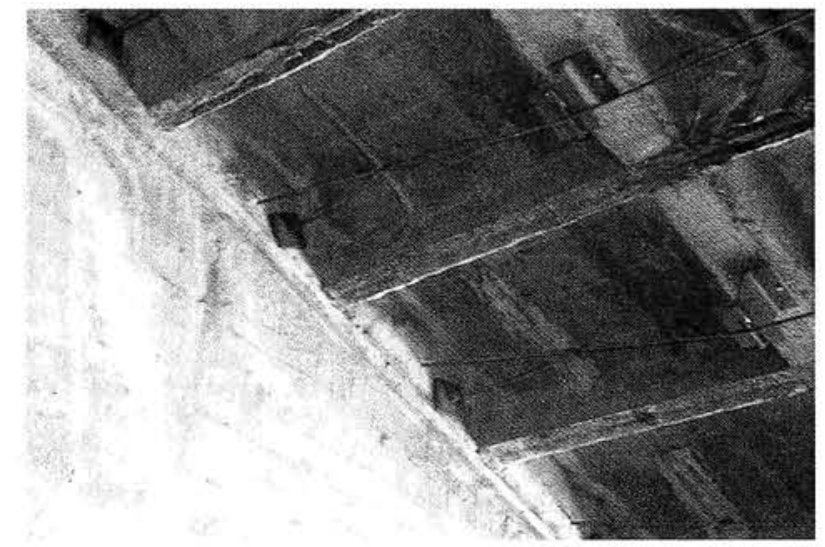

Foto 5.-Placa de anclaje y condena del tensado.

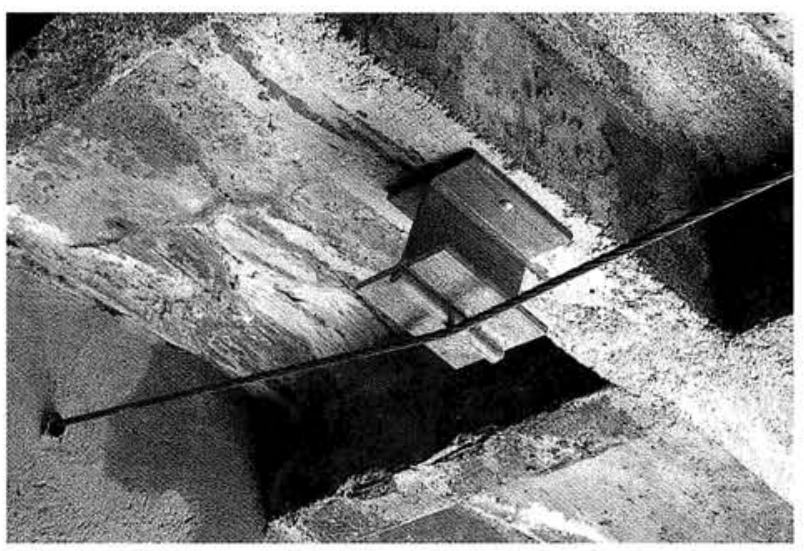

Foto 6.-Apoyo del cableado en pieza de apeo.

- Diámetro $12,7 \mathrm{~mm}$.

- Límite elástico $16.900 \mathrm{~kg}$.

- Carga de rotura $18.800 \mathrm{~kg}$.

- Coeficiente de minoración de hormigón $=1,50$.

- Coeficiente de mayoración de cargas $=1,60$.

Sobre estas previsiones se efectuaron hasta 8 distintas hipótesis de cálculo, abarcando toda la gama de posibilidades y realizándose en cada una de ellas las oportunas comprobaciones en ordenador.

De común acuerdo todos los técnicos actuantes dieron el visto bueno a las medidas de seguridad adoptadas, habiendo funcionado la totalidad de las instalaciones con posterioridad a plena satisfacción, si bien los cambios climatológicos y la humedad ambiente hacen necesaria la periódica supervisión del sistema, que siendo una medida provisional, de ningún modo definitiva, exige, en un plazo prudencial de tiempo, una actuación más enérgica y definitiva. 